\title{
Indicadores de gestión de mantenimiento en empresas de servicio petrolero
}

\author{
Maintenance management indicators in oil service companies
}

\author{
Marle Cecilia Martínez Ramírez \\ marlemartinez@yahoo.es \\ ORCID 0000-0002-1531-3320 \\ Universidad del Zulia
}

\section{Dilú Virginia Carbonell Soto \\ dilucarbonell@gmail.com \\ ORCID 0000-0001-8394-7790 \\ Universidad del Zulia}

Artículo recibido febrero 2020 | Arbitrado en marzo 2020 | Publicado en mayo 2020

\section{RESUMEN}

El objetivo de la investigación fue determinar los indicadores de gestión de mantenimiento que utilizan las empresas de servicio petrolero ubicadas en la Costa Oriental del Lago de Maracaibo. La investigación fue de tipo analítico, con un diseño no experimental, de campo, transeccional. La población estuvo constituida por veintiséis empresas, cuyas unidades informantes fueron los gerentes generales. La técnica aplicada fue la encuesta, el instrumento de recolección de datos fue un cuestionario. Se estimó la confiabilidad mediante el Coeficiente Alfa Cronbach cuyo resultado fue de 0,91; muy alta confiabilidad. El análisis de los datos se llevó a cabo mediante la estadística descriptiva, utilizando la media aritmética y la desviación estándar. Finalmente se evidenció un moderado uso de los indicadores considerados en esta investigación, lo cual devela la necesidad de revisar detalladamente las ventajas y desventajas del uso frecuente de cada uno de ellos por parte de las empresas analizadas.

Palabras clave: Confiabilidad; costos; disponibilidad; indicadores de gestión de mantenimiento; mantenibilidad

\section{ABSTRACT}

The objective of the investigation was to determine the maintenance management indicators used by the oil service companies located on the eastern shore of Lake Maracaibo. The research was analytical, with a nonexperimental, field, transectional design. The population consisted of twenty-six companies, whose reporting units were general managers. The applied technique was the survey, the data collection instrument was a questionnaire. Reliability was estimated using the Alpha Cronbach Coefficient whose result was 0.91; very high reliability. Data analysis was carried out using descriptive statistics, using the arithmetic mean and standard deviation. Finally, a moderate use of the indicators considered in this research was evident, which reveals the need to review in detail the advantages and disadvantages of the frequent use of each of them by the companies analyzed.

Key words: Reliability; costs; availability; maintenance management indicators; maintainability 


\section{INTRODUCCIÓN}

Para el mundo de los negocios, conducir bien una organización supone decidir correctamente. Pero la decisión en sí misma es producto de un proceso de reflexión que involucra diversas actividades. Esta reflexión sistemática, ordenada, abarca todas las gestiones que se desarrollan en las empresas, en la búsqueda de una integración global, estratégica y operativa.

Las organizaciones empresariales en los últimos tiempos están llamadas a ser altamente competitivas, productivas, de alto desempeño frente a un entorno completamente dinámico y lleno de incertidumbre, razón por la cual están obligadas a gestionar sus recursos de manera efectiva, contar con personal altamente capacitado, apoyados en normativas organizacionales que contribuyan al mayor rendimiento productivo, reducir el tiempo en la ejecución de procesos, minimizando costos de operaciones y sobre todo, garantizando a sus clientes el abastecimiento oportuno de los productos o compromisos contraídos.

Al respecto, son muchos los cambios que están ocurriendo en el ámbito mundial en materia económica, exigiendo una nueva postura por parte de las organizaciones empresariales, provocando una rápida inestabilidad si no se está preparado gerencialmente hablando para el diseño de metodologías y ajustarse a las novedades, modalidades y exigencias de los mercados cambiantes de la actualidad. Estos cambios producidos en el siglo XXI van en continuo aumento, no tienen precedentes, constituyen transformaciones permanentes, aceleradas, e interdependientes en los ámbitos: industrial, científico y tecnológico, por lo cual, las organizaciones empresariales deben adaptándose a las nuevas exigencias.

Estos planteamientos aplican a todo tipo de empresas, por ello para competir en un mundo dinámico y globalizado las empresas están obligadas a diferenciar sus productos, generar estrategias competitivas estimulando la optimización de los recursos disponibles vinculando e integrando todas sus áreas.

En función de las ideas expuestas, las empresas venezolanas no escapan de esta realidad, tal es el caso de las pertenecientes al sector servicio petrolero de la Costa Oriental del Lago de Maracaibo, cuyas actividades ejecutadas principalmente son: trabajos de limpieza e inyección de pozos, servicio a equipos de perforación, trabajos civiles, diques, drenajes, reparación de motores de balancines y bombas, electromecánica, entre otras. Las mencionadas empresas aplican los indicadores de gestión a fin de conocer las especificaciones establecidas por la ingeniería del producto y proporcionar asistencia al departamento de fabricación, con el objetivo de cumplir ciertas especificaciones. Por tanto, los indicadores de gestión ofrecen una estrategia para asegurar el mejoramiento continuo de la calidad.

Con base a lo anterior, cabe resaltar que las empresas de servicio petrolero encaminan sus actividades en mantener la competitividad, satisfacción del cliente, bajos costos, conllevando que el producto/servicio se distribuya con tiempos de entrega que no eleven los costos. Si bien es cierto cada empresa contempla su misión, visión y estrategias, sin embargo a partir de la observación directa y entrevistas no estructuradas 
realizadas no se aplican los indicadores de gestión de mantenimiento pese a los esfuerzos realizados, como consecuencia se evidencian paradas no planificadas, averías imprevistas en la maquinaria o equipo, falta de cumplimiento en las fechas de entregas, ausencia de mecanismos de control, fallas no reportadas, mantenimiento no ejecutado a tiempo.

De lo expresado anteriormente, el objetivo que orienta la investigación es determinar los indicadores de gestión de mantenimiento que utilizan las empresas de servicio petrolero de la Costa Oriental del Lago a fin de alcanzar un cumplimiento tanto efectivo como eficiente de los objetivos establecidas en dichas empresas.

\section{Indicadores de gestión}

Según Mora (2011) uno de los factores determinantes para que todo proceso se lleve a cabo con éxito, es implementar un sistema adecuado de indicadores para medir la gestión de los mismos, con el fin de que se puedan implementar indicadores en posiciones estratégicas que reflejen un resultado óptimo en el mediano y largo plazo, que permita medir las diferentes etapas del proceso productivo.

En relación a este tópico, para Heredia (2013) la existencia de indicadores de gestión o ratios de apoyo, desarrollados de modo individual por cada departamento, permiten evaluar el resultado de sus actividades, para su eventual conocimiento y utilización local por el departamento que lo desarrolló. Bajo el enfoque de Heredia, lo más importante de los indicadores es el seguimiento de su evolución en el tiempo.

Ahora bien, Mora (2011) considera que cada empresa, y según el tipo de contabilidad elegida, tendrá sus propios índices, los más adecuados al tipo de gestión establecida, con respecto a los elementos básicos de la organización, permite resumirlo en: planificación, aprovisionamiento, gestión de inventarios o existencias, gestión de almacenes, gestión de pedidos y distribución, así como el servicio al cliente. Asimismo,

Con el objeto de constatar si el desempeño organizacional es el más adecuado, se realizan mediciones de los procesos al fin de llevar a cabo un control (lo que no se mide no se controla), el interés de verificar que las acciones se realiza dentro de los parámetros preestablecidos, y que se están tomando las decisiones más acertadas, es decir, que se está llevando a cabo una adecuada gestión.

Según Kaplan y Norton (2002), afirman que un indicador es la medida gerencial que permite la evaluación del desempeño de las labores en los sistemas productivos frente a los objetivos, en la cual relacionan actividades, estrategia, responsabilidades, es decir, los indicadores activan en todo sistema de producción, ellos son la base para verificar la actividad económica y sus resultados en cuanto a los costos, la calidad del servicio o producto.

\section{Indicadores de gestión de mantenimiento}

En el ámbito del mantenimiento los autores Jiménez y Milano (2006), expresan que existen una variedad de indicadores que se pueden utilizar en la gestión del mantenimiento, estos se implementan para medir la gestión de mantenimiento de sistemas productivos, los cuales son utilizados para cumplir con las metas definidas en un plan de trabajo generalmente realizado anualmente. Previamente se deben llevar registros de datos que permitan el cálculo periódico de los indicadores. 
En ese orden de ideas, Leal y Zambrano (2011), consideran que la función del mantenimiento debe estar en una continua evaluación para lograr la optimización del mismo y una forma de encontrarlo es mediante la generación, aplicación y evaluación de los indicadores de gestión. Esto con el fin de llevar un control que permita a la empresa, minimizar los tiempos de entrega para aumentar la satisfacción del cliente y que por ende traerá como consecuencia, mayor margen de ganancia o beneficio a través de mínimos costos operacionales.

Por su parte, Hernández y Navarrete (2005) acotan que los indicadores más usuales que se emplean en un departamento de mantenimiento son: disponibilidad, confiabilidad, mantenibilidad y costos. Así, la disponibilidad permite medir que los equipos estén operativos cuando se necesiten. Mientras que la confiabilidad se refiere a que los equipos no fallen mientras se usan. La mantenibilidad indica la probabilidad de que el equipo sea restaurado a sus condiciones normales de funcionabilidad. Finalmente el indicador de costo ayuda a establecer programas de reducción de costos necesarios en la gestión de mantenimiento.

Con base a las consideraciones anteriores, es importante señalar que para cualquier actividad de mantenimiento es necesario definir una serie de indicadores que permitan el seguimiento de la gestión de mantenimiento frente al sistema de producción; la información definida por los indicadores cuantifica la eficacia y la eficiencia de dichas actividades, de este modo se evalúa de forma objetiva la gestión.

\section{Disponibilidad}

Para Souris (2010), la disponibilidad, objetivo principal del mantenimiento, puede ser definida como la confianza de que un componente o sistema que sufrió mantenimiento, ejerza su función satisfactoriamente para un tiempo dado. En la práctica, la disponibilidad se expresa como el porcentaje de tiempo en que el sistema está listo para operar o producir, esto en sistemas que operan continuamente.

De acuerdo con García (2013) es definida como la probabilidad que un sistema, subsistema o equipo esté disponible para su uso durante un tiempo dado; depende del sistema técnico, la eficiencia y eficacia de mantenimiento. La disponibilidad es función o parámetro que se trabaja en conjunto con la confiabilidad y Mantenibilidad.

En este sentido permite conocer que la disponibilidad de los equipos e instalaciones sea la adecuada para lograr las metas del mantenimiento. Para ser evidenciada debe prever un análisis, o metodología que permita establecer la jerarquía o prioridades de procesos, sistemas y equipos, creando una estructura que facilite la toma de decisiones para que estas sean acertadas y efectivas, direccionando el esfuerzo y recursos en áreas donde sea más importante mejorar la disponibilidad, basado en la realidad actual.

También destaca Ferrer (2009) que una vez identificadas estas zonas, es mucho más fácil diseñar una estrategia, para realizar estudios que mejoren la disponibilidad operacional, iniciando las aplicaciones en el conjunto de procesos o elementos que formen parte de la zona de alta criticidad. Los criterios para realizar un 
análisis de disponibilidad están asociados con: seguridad, ambiente, producción, costos de operación y mantenimiento, datos de fallas y tiempo de reparación principalmente.

Los periodos de tiempos nunca incluyen las paradas planificadas ni paros por producción, solo se toma en cuenta los tiempos relacionados con falla de equipos. La disponibilidad da un valor entre 0 y $1, y$ mientras más cercano al 1 es mejor, por lo tanto se puede mejorar el valor de la disponibilidad mejorando la confiabilidad (aumentando el tiempo promedio de fallas) o mejorando la mantenibilidad (es decir disminuyendo el tiempo promedio de reparación).

En base a los planteamientos anteriores, las investigadoras definen que la disponibilidad de un equipo o sistema es un parámetro que indica cuánto tiempo está funcionando ese equipo o sistema de producción, respecto de la duración total durante la que se hubiese deseado su funcionamiento.

\section{Confiabilidad}

Para Duffuaa y col. (2014), la confiabilidad representa una medida de la frecuencia de una falla, definida como el tiempo de operación entre número de fallas. Por su parte Rodríguez (2008), la define como la probabilidad que un equipo o sistema de producción, desarrolle su función bajos condiciones específicas, durante un periodo de tiempo determinado, conocido también como fiabilidad.

Cabe destacar, por lo antes mencionado que la confiabilidad de un equipo o sistema, es directamente proporcional a la aplicación de un efectivo mantenimiento y al diseño apropiado para cumplir con las especificaciones del sistema de producción, en este sentido, al no realizar mantenimiento y/o poseer un diseño no acorde a los requerimientos de producción, éste poseerá una baja confiablidad.

Asimismo Tavares (2006), define la confiabilidad como la probabilidad de que un componente o equipo fallara estando en servicio durante un periodo determinado cuando es operado en condiciones razonablemente uniformes de presión, temperatura, velocidad, vibraciones, entre otras. Un equipo es confiable cuando funciona cada vez que se necesita y hace bien el trabajo para el cual fue diseñado, de otra manera se dice que es desconfiable.

Por su parte, según Hernández y Navarrete (2005) la fiabilidad o confiabilidad se define como la probabilidad de que un bien funcione adecuadamente durante un período determinado bajo condiciones operativas específicas (por ejemplo, condiciones de presión, temperatura, velocidad, tensión o forma de una onda eléctrica, nivel de vibraciones, entre otras). Para Leal $y$ Zambrano (2011), es la probabilidad de que un elemento o sistema de producción realizará su función prevista sin fallas o averías, en un período de tiempo especificado bajo condiciones dadas de operación. En otras palabras, la confiabilidad de un equipo o sistema es la probabilidad de que éste opere sin ningún contratiempo, al ser requerido por el sistema de producción.

Además, Duffuaa y col. (2014), consideran que en la medida que la organización adopte la confiabilidad del mantenimiento, toma ciertas consideraciones que favorecen al enfoque para la administración de los activos, en 
donde se puede resaltar lo siguiente: a) la frecuencia de fallas prematuras puede reducirse mediante una lubricación adecuada; b) si la falla no puede prevenirse, la inspección y la medición periódica pueden ayudar a reducir la severidad de la falla y el posible efecto dominó en otros componentes del sistema del equipo; c) en donde se pueda vigilar la degradación gradual de una función o un parámetro, como la calidad de un producto o la vibración de una máquina, puede detectarse el aviso de una falla inminente.

Desde la posición de las investigadoras, la confiabilidad del mantenimiento es lo que se planifica y programa con el objetivo de ajustar, reparar o cambiar partes en equipos antes de que ocurra la falla o daños mayores, eliminando o reduciendo al mínimo los gastos de mantenimiento, es decir, que es necesario establecer controles de gestión con la finalidad de aumentar la productividad.

\section{Mantenibilidad}

Según Mesa y col. (2006) la mantenibilidad describe el tiempo promedio de reparación, es decir la aplicación de la acción correctiva sobre las fallas, pero dicho tiempo incluye factores que inciden directamente sobre éste. En este sentido se describen algunos, tal como lo son la mano de obra capacitada, la planificación de las actividades de mantenimiento (planificación y programación), la disponibilidad de materiales y repuestos, y la accesibilidad para aplicar el mantenimiento. Todos estos factores determinan la mantenibilidad, por lo que se debe considerar los tiempos que estos desempeñan y sumárselos a la ejecución propiamente dicha de la actividad.

Para Torres (2010), la mantenibilidad es la probabilidad de que una máquina, equipo o un sistema pueda ser reparado a una condición especificada en un período de tiempo dado, en tanto su mantenimiento sea realizado de acuerdo con ciertas metodologías y recursos determinados con anterioridad. Análogamente Nava (2009), sostiene que es la probabilidad de que el equipo sea restaurado a sus condiciones normales de funcionabilidad, luego de la aparición de la falla, bajo condiciones preestablecidas de mantenimiento.

En ese orden de ideas Tavares (2006), afirma que la mantenibilidad es un parámetro estadístico que se define como la probabilidad que tiene un ítem en estado de falla, a ser diagnosticado y reparado con éxito en un tiempo y en el contexto de operación establecido. Visto así, es la facilidad de un ítem de ser mantenido, además, es la característica inherente de un elemento, asociada a su capacidad de ser recuperado para el servicio cuando se realiza la tarea de mantenimiento.

Para Rodríguez (2008), la mantenibilidad es la probabilidad de que un equipo en estado de falla sea restablecido a una condición determinada de operación en un período de tiempo, utilizando los recursos necesarios. Por su parte, Nava (2009) explica que es la probabilidad de que el equipo sea restaurado a sus condiciones normales de funcionabilidad, luego de la aparición de la falla, bajo condiciones preestablecidas de mantenimiento.

Es decir, la mantenibilidad es un indicador que mide el tiempo de reparación 
del equipo y la velocidad del mantenimiento, siempre bajo condiciones óptimas de mantenimiento, sin riesgos para el personal y el ambiente.

Es común analizar la mantenibilidad dependiendo del tipo de falla ocurrida; en algunos casos la falla origina una mantenibilidad menor que en otros. Recordando que a mayor mantenibilidad menor tiempo de ejecución de los trabajos y viceversa.

Es importante destacar, como explica García (2011), que la mantenibilidad sobre los sistemas de control, no hace parte de las definiciones corporativas en relación con el mantenimiento de infraestructura, de igual manera, tampoco se encuentra soportado en las plataformas de mantenimiento. Es probable que esta situación se relacione con el bajo índice de fallas a lo largo de la vida útil de los sistemas de control.

En este punto, los tres objetivos principales que cumplen los sistemas de control en la mantenibilidad son: garantizar la seguridad del proceso; asegurar la operabilidad, es decir, garantizar que los flujos y variables del mismo se mantengan en los valores deseados y en los rangos de operación, controlar la calidad del producto, el consumo de energía del proceso. De tal forma, los departamentos encargados consciente de la criticidad de equipos y sistemas de control en las operaciones, han visto la necesidad de someterlos a análisis concienzudo desde el punto de vista de la confiabilidad, disponibilidad y mantenibilidad que les permitan orientar las acciones de mantenimiento.

Para las investigadoras, la mantenibilidad es la probabilidad que el equipo sea restaurado a sus condiciones normales de funcionabilidad, luego de la aparición de la falla, bajo condiciones preestablecidas de mantenimiento. Es decir, la mantenibilidad es un indicador que mide el tiempo de reparación del equipo y velocidad del mantenimiento, siempre bajo condiciones óptimas de mantenimiento, ejecutado sin riesgos para el personal y el ambiente.

\section{Costos}

El costo como indicador de la gestión de mantenimiento, es un índice económico que permite el seguimiento de la evolución de los resultados internos y cierta comparación del mantenimiento entre diferentes plantas. Autores como Rodríguez (2008) considera que las medidas de entrada se relacionan directamente con el costo de realizar el mantenimiento, y que algunos de los factores importantes a considerar son: mano de obra, materiales, contrato, servicios de taller, alquiler de equipos, herramientas, gastos generales de mantenimiento, gastos generales de la empresa o la planta, incluyendo la depreciación.

Junto a los costos de las categorías anteriores de medidas de entrada tal vez haya medidas específicas no relacionadas con costos para cada una de ellas. El autor citado menciona como ejemplos; inversión en inventarios, rotación de inventario, nivel de servicio, faltantes u obsolescencias del inventario.

Según Molina (2015) para tomar decisiones basadas en la estructura de costos, y teniendo presente que una de las principales tareas es minimizar los costos, entonces es importante conocer sus componentes. Duffuaa y col. (2014), describen que la información de los costos debe estar incluida en las órdenes de trabajo, y que esta debe reflejar un resumen 
mensual de los costos de mantenimiento. El indicador del costo ayuda a establecer programas de reducción de costos necesarios en la gestión de mantenimiento.

En ese mismo orden de ideas Martínez (2007), considera que desde el punto de vista de la organización de mantenimiento el reporte de los costos debe servir a los siguientes requisitos específicos: la supervisión del personal que labora en los mantenimientos la cual necesita medir efectividad; la gerencia de mantenimiento requiere observar la tendencia de los costos de mantenimiento, sin afectar la calidad y la producción; por último la gerencia general debe conocer los costos relacionados con el mantenimiento, los cuales son esenciales para calcular los costos de producción.

Según Jiménez y Milano (2006), el elemento de costo que antes no era tan importante en el mantenimiento de los equipos, hoy en día se ha convertido en la prioridad de control más importante en las empresas sistemas de producción. Es por eso que consideran el costo como indicador para la evaluación de los sistemas de gestión de mantenimiento.

De tal manera, señala Ferrer (2009), se debe tener en cuenta dentro de los costos el mantenimiento correctivo, como parte fundamental de la gestión de mantenimiento, pues será consecuencia de las averías imprevistas en la maquinaria, como de las reparaciones programadas por otros tipos de mantenimiento a la maquinaria.

Al respecto destaca este mismo autor que, parecería imposible reducir este tipo de gasto de mantenimiento, dado que viene directamente de la necesidad de realizar una reparación para continuar produciendo o prestando el servicio. La manera de reducir este tipo de gasto no pasa por dejar de hacer mantenimiento correctivo, sino por evitar que se produzcan las averías inesperadas.

Desde el punto de vista de las investigadoras, el reporte de los costos de mantenimiento debe servir a los siguientes requisitos específicos: supervisión del personal que labora en el mantenimiento la cual necesita medir su efectividad; la gerencia de mantenimiento requiere observar la tendencia de los costos, con el detalle necesario para atender las áreas de atención especial y así lograr reducir los costos de mantenimiento, sin afectar la calidad y la producción; por último, la gerencia debe conocer los costos relacionados con el mantenimiento, los cuales son esenciales para calcular los costos de producción.

\section{MÉTODO}

En virtud de lograr el propósito del presente estudio, se procedió a establecer el tipo de investigación como descriptiva con enfoque cuantitativo. En concordancia con lo anterior, Hurtado (2015) establece que éstas buscan exponer el evento estudiado, haciendo una enumeración detallada de sus características.

Por otra parte, el diseño de investigación, de acuerdo con Arias (2016) es la estrategia general que adopta el investigador para responder al problema planteado. En ese mismo sentido, es un plan sistemático y alcanzable, elaborado en función de aplicar estrategias para la recolección y análisis de los datos. Por lo anteriormente expuesto, en el caso puntual del presente estudio, se ha establecido un diseño de campo, no experimental, transeccional.

Para realizar este planteamiento, se considera que el diseño de campo, es 
descrito por Hurtado (2015) como aquella que permite observar y recolectar datos de fuentes vivas en su ambiente natural. Se refiere que el diseño de la investigación hace explícitos los aspectos operativos de la misma, esta se define con base al procedimiento, corresponde a la estructura y a la forma como la investigación es realizada, a la manera como la indagación es concebida, a fin de obtener respuestas a las interrogantes planteadas.

Conforme con la definición de los autores antes mencionados, se deduce que la investigación se ajusta perfectamente al diseño de campo puesto al observar directamente los factores que se estudiaran. En este sentido, los datos que se utilizan para obtener la información son tomados directamente de los escenarios y realidades, en este caso las empresas de servicio petrolero.

Una vez establecido el diseño de la investigación, se procedió a definir la población objeto de estudio y por ende, serán los entes sobre los cuales se aplicarán los instrumentos de recolección de datos. En ese sentido, para Arias (2016), es un conjunto finito o infinito de elementos con características comunes para los cuales, serán extensivas las conclusiones de la investigación.

Considerando lo antes expuesto, la técnica utilizada en el presente estudio fue la encuesta y el instrumento de recolección de datos utilizado fue el cuestionario estructurado con proposiciones afirmativas de escala de frecuencia (Siempre, Casi Siempre, Algunas Veces, Casi Nunca y Nunca), estructurado en 20 ítems. En este orden de ideas la población estuvo constituida por veintiséis empresas de servicio petrolero de los Municipios Lagunillas y Cabimas activas para la fecha, cuyas unidades informantes fueron los gerentes generales.

Seguidamente, se procedió con la aplicación de los criterios de validez y confiabilidad. Estos permiten que una vez formulados los ítems pertinentes del instrumento de recolección de datos, se sometan a un estudio técnico para la definir la validez del mismo en referencia a la investigación planteada. En tal sentido, para Hernández y col. (2014) sostienen que la validez es el grado en el que un instrumento en verdad mide lo que se pretende medir. De acuerdo con lo anterior, esta permite establecer seguridad al utilizar un instrumento validado, se estará seguro que el mismo es confiable y permite medir adecuadamente el fenómeno de estudio.

Así, la validación del instrumento diseñado en esta investigación, se realizó a través de cinco (5) expertos, quienes aportaron sugerencias sobre los ítems diseñadas referentes a los indicadores de gestión de mantenimiento. Ahora bien, para determinar la confiabilidad del instrumento de recolección de datos, se aplicó el coeficiente Alfa Cronbach, obteniéndose un coeficiente de 0,92 lo cual, de acuerdo al criterio de Hernández y col. (2014), se considera un instrumento de muy alta confiabilidad.

Con respecto a las técnicas de análisis de datos utilizadas en la presente investigación, se debe indicar que se recurrió a la estadística descriptiva, utilizando la media aritmética y el baremo aplicado se muestra en el cuadro 1. 
Cuadro 1. Baremo para la interpretación de la media aritmética

\begin{tabular}{|c|c|c|c|c|}
\hline \multirow{6}{*}{$\begin{array}{l}\text { Indicadores } \\
\text { de la gestión de } \\
\text { mantenimiento }\end{array}$} & Alternativa & Rango & Intervalo & Categoría \\
\hline & Siempre & 5 & $4.21-5$ & $\begin{array}{l}\text { Muy alto uso del indicador en la } \\
\text { gestión }\end{array}$ \\
\hline & Casi siempre & 4 & $3.41-4.20$ & Alto uso del indicador en la gestión \\
\hline & Algunas veces & 3 & $2.61-3.40$ & $\begin{array}{l}\text { Moderado uso del indicador en la } \\
\text { gestión }\end{array}$ \\
\hline & Casi nunca & 2 & $1.81-2.60$ & Bajo uso del indicador en la gestión \\
\hline & Nunca & 1 & $1-1.80$ & $\begin{array}{l}\text { Muy bajo uso del indicador en la } \\
\text { gestión }\end{array}$ \\
\hline
\end{tabular}

Con respecto a la medida de variabilidad, se empleó la desviación estándar, la cual indica el grado de dispersión de las respuestas y su nivel de confiabilidad, en función de la escala de medición utilizada, tal como se muestra en el cuadro 2.

Cuadro 2. Baremo para la interpretación de la desviación estándar

\begin{tabular}{|c|c|}
\hline Intervalo & Descripción \\
\hline $1,61-2,00$ & $\begin{array}{l}\text { Muy alta dispersión de las respuestas y, muy baja confiabilidad de las } \\
\text { mismas. }\end{array}$ \\
\hline $1.21-1,60$ & Alta dispersión de las respuestas y baja confiabilidad de las mismas. \\
\hline $0,81-1,20$ & $\begin{array}{l}\text { Moderada dispersión de las respuestas y, moderada confiabilidad de las } \\
\text { mismas. }\end{array}$ \\
\hline $0.41-0,80$ & Baja dispersión de las respuestas y alta confiabilidad de las mismas. \\
\hline $0-0.4$ & $\begin{array}{l}\text { Muy baja dispersión de las respuestas y, muy alta confiabilidad de las } \\
\text { mismas. }\end{array}$ \\
\hline
\end{tabular}

\section{RESULTADOS Y DISCUSIÓN}

A continuación en la tabla 1 se determinan los indicadores de gestión de mantenimiento que utilizan las empresas de servicio petrolero ubicadas en la Costa Oriental del Lago de Maracaibo, la cual se desglosó en cuatro (4) indicadores, a saber: disponibilidad, confiabilidad, mantenibilidad y costos; una vez aplicado del cuestionario, se logró obtener la información necesaria que permitió demostrar los siguientes resultados:
Como se puede observar, en la tabla 1 , los resultados obtenidos de la aplicación del instrumento demuestran el comportamiento del indicador disponibilidad, el cual presenta una media aritmética de 3,35; indicando un moderado uso del indicador disponibilidad en la gestión de mantenimiento de las empresas de servicio petrolero de la Costa Oriental del Lago. 
Al mismo tiempo, este indicador alcanzó una desviación estándar de 0,99 lo que revela una moderada dispersión en las respuestas dadas por los encuestados, así como una moderada confiabilidad de las mismas.

Tabla 1. Resultados indicador disponibilidad.

\begin{tabular}{|c|c|c|c|c|c|c|c|c|c|c|}
\hline № ítem & \multicolumn{2}{|c|}{1} & \multicolumn{2}{|c|}{2} & \multicolumn{2}{|r|}{3} & \multicolumn{2}{|c|}{4} & \multicolumn{2}{|r|}{5} \\
\hline $\begin{array}{l}\text { Descripción } \\
\text { Ítem }\end{array}$ & \multicolumn{2}{|c|}{$\begin{array}{l}\text { La } \\
\text { disponibilidad } \\
\text { de los equipos } \\
\text { es la adecuada } \\
\text { para lograr las } \\
\text { metas del } \\
\text { mantenimiento. }\end{array}$} & \multicolumn{2}{|c|}{$\begin{array}{l}\text { Se registra la } \\
\text { disponibilidad } \\
\text { real de } \\
\text { equipos. }\end{array}$} & \multicolumn{2}{|c|}{$\begin{array}{l}\text { La } \\
\text { organización } \\
\text { mide su } \\
\text { desempeño } \\
\text { a través de } \\
\text { indicadores } \\
\text { para medir } \\
\text { el } \\
\text { desempeño. }\end{array}$} & \multicolumn{2}{|c|}{$\begin{array}{l}\text { Se determina } \\
\text { la } \\
\text { disponibilidad } \\
\text { funcional de } \\
\text { los equipos. }\end{array}$} & \multicolumn{2}{|c|}{$\begin{array}{l}\text { Se realizan } \\
\text { mediciones } \\
\text { sobre la } \\
\text { probabilidad } \\
\text { de que un } \\
\text { equipo se } \\
\text { encuentre } \\
\text { operativo } \\
\text { cuando se } \\
\text { requiera su } \\
\text { uso. }\end{array}$} \\
\hline Opción & $\mathbf{F a}$ & Fr & $\mathbf{F a}$ & Fr & $\mathbf{F a}$ & Fr & $\mathbf{F a}$ & Fr & $\mathbf{F a}$ & Fr \\
\hline$S$ & 2 & 6,67 & 7 & 23,33 & 9 & 30,00 & 3 & 10,00 & 2 & 6,67 \\
\hline CS & 9 & 30,00 & 9 & 30,00 & 7 & 23,33 & 6 & 20,00 & 7 & 23,33 \\
\hline AV & 14 & 46,67 & 10 & 33,33 & 8 & 26,67 & 16 & 53,33 & 14 & 46,67 \\
\hline $\mathrm{CN}$ & 4 & 13,33 & 4 & 13,33 & 4 & 13,33 & 5 & 16,67 & 5 & 16,67 \\
\hline $\mathbf{N}$ & 1 & 3,33 & 0 & 0,00 & 2 & 6,67 & 0 & 0,00 & 2 & 6,67 \\
\hline Total & 30 & 100,00 & 30 & 100,00 & 30 & 100,00 & 30 & 100,00 & 30 & 100,00 \\
\hline Media ítem & \multicolumn{2}{|c|}{3,23} & \multicolumn{2}{|c|}{3,63} & \multicolumn{2}{|c|}{3,57} & \multicolumn{2}{|c|}{3,23} & \multicolumn{2}{|c|}{3,07} \\
\hline Categoría & \multicolumn{2}{|c|}{ Moderado uso } & \multicolumn{2}{|c|}{ Alto uso } & \multicolumn{2}{|c|}{ Alto uso } & \multicolumn{2}{|c|}{ Moderado uso } & \multicolumn{2}{|c|}{$\begin{array}{l}\text { Moderado } \\
\text { uso }\end{array}$} \\
\hline $\begin{array}{l}\text { Media } \\
\text { Indicador }\end{array}$ & \multicolumn{10}{|c|}{3,35} \\
\hline Categoría & \multicolumn{10}{|c|}{ Moderado uso } \\
\hline Desviación & \multicolumn{10}{|c|}{0,99} \\
\hline Categoría & \multicolumn{10}{|c|}{ Moderada dispersión / Moderada confiabilidad } \\
\hline
\end{tabular}

Por otro lado, al analizar detalladamente los resultados obtenidos para cada uno de los ítems del indicador, es de notarse que los aspectos relacionados con los ítems 2 y 3 , alcanzaron la categoría de alto uso de este indicador de gestión en la medición de su desempeño, presentando medias aritméticas de 3,63; y 3,57; respectivamente. Relacionados al registro de la disponibilidad real de los equipos y la utilización de indicadores de gestión para medir su desempeño.

No obstante, es conveniente destacar que, de acuerdo a los encuestados, existe ciertos aspectos en el uso del indicador disponibilidad en las empresas de servicio petrolero de la Costa Oriental del Lago, con el cual no están del todo conformes, siendo 
los casos específicos de los ítems 1, 4 y 5 , concernientes a: en el ítem 1 al consultarles si la disponibilidad de los equipos es la adecuada para lograr las metas del mantenimiento, un $46,67 \%$ del total de los encuestados se inclinó por la alternativa algunas veces y un $30 \%$ opina que casi siempre, lo que evidencia su inconformidad al respecto. De igual manera, la media aritmética obtenida por este ítem $(3,23)$ lo ubica dentro de la categoría de moderado uso, en función del baremo de interpretación correspondiente.

En el caso del ítem 4, donde se les consulta si determinan la disponibilidad funcional de los equipos de acuerdo a los requerimientos del mantenimiento, un $53,33 \%$ manifestó que algunas veces, mostrándose para este ítem una media de 3,23 que lo ubica en la categoría de moderado uso.

Para el ítem 5, la consulta estuvo dirigida a conocer con qué frecuencia se realizan mediciones sobre la probabilidad de que un equipo se encuentre operativo cuando se requiera su uso, a lo cual el $46,67 \%$ respondió que algunas veces, determinándose una media de 3,07, indicando un moderado uso de esta actividad en la gestión de mantenimiento que se analiza.

En la tabla 2, se exhiben los resultados obtenidos para el indicador confiabilidad, el cual ostenta una media aritmética de 3,19 evidenciando que, según la apreciación de los encuestados, las empresas de servicio petrolero de la Costa Oriental del Lago estudiadas demuestran un moderado uso de este indicador de gestión en la medición del desempeño, en relación a las actividades, necesarias para su medición. A la vez, este indicador presenta una desviación estándar de 1,02 indicando una moderada dispersión de las respuestas dadas por los encuestados y una moderada confiabilidad de las mismas.

Se evidencia un alto uso para los ítems 2 y 3 , referidos a la confiabilidad y funcionabilidad de los equipos, respectivamente, alcanzando medias de 3,47 en ambos casos. Estos resultados permiten afirmar que existe un alto uso de los indicadores para medir si los equipos utilizados en las operaciones de la gerencia son confiables, así como para el monitoreo de que los equipos operan sin fallas durante un tiempo previsto de funcionabilidad. 
Tabla 2. Resultados indicador confiabilidad.

\begin{tabular}{|c|c|c|c|c|c|c|c|c|c|c|}
\hline № ítem & \multicolumn{2}{|r|}{1} & \multicolumn{2}{|r|}{2} & \multicolumn{2}{|c|}{3} & \multicolumn{2}{|r|}{4} & \multicolumn{2}{|r|}{5} \\
\hline $\begin{array}{l}\text { Descripción } \\
\text { Ítem }\end{array}$ & \multicolumn{2}{|c|}{$\begin{array}{l}\text { La frecuencia } \\
\text { con que } \\
\text { fallan los } \\
\text { equipos se } \\
\text { puede } \\
\text { considerar } \\
\text { adecuada. }\end{array}$} & \multicolumn{2}{|c|}{$\begin{array}{l}\text { Los equipos } \\
\text { utilizados en } \\
\text { las } \\
\text { operaciones } \\
\text { de la empresa } \\
\text { son } \\
\text { confiables. }\end{array}$} & \multicolumn{2}{|c|}{$\begin{array}{l}\text { Los equipos } \\
\text { operan sin fallas } \\
\text { durante un } \\
\text { tiempo previsto } \\
\text { de su } \\
\text { funcionamiento. }\end{array}$} & \multicolumn{2}{|c|}{$\begin{array}{l}\text { Se llevan } \\
\text { registros } \\
\text { relacionados } \\
\text { con la } \\
\text { confiabilidad } \\
\text { de los } \\
\text { equipos. }\end{array}$} & \multicolumn{2}{|c|}{$\begin{array}{l}\text { Se elaboran } \\
\text { históricos de } \\
\text { fallas de un } \\
\text { equipo cada } \\
\text { momento de } \\
\text { su vida útil. }\end{array}$} \\
\hline Opción & $\mathbf{F a}$ & Fr & $\mathbf{F a}$ & Fr & $\mathbf{F a}$ & $\mathbf{F r}$ & $\mathbf{F a}$ & Fr & $\mathbf{F a}$ & $\mathbf{F r}$ \\
\hline $\mathbf{S}$ & 1 & 3,33 & 3 & 10,00 & 3 & 10,00 & 3 & 10,00 & 4 & 13,33 \\
\hline CS & 6 & 20,00 & 11 & 36,67 & 12 & 40,00 & 8 & 26,67 & 11 & 36,67 \\
\hline AV & 9 & 30,00 & 14 & 46,67 & 11 & 36,67 & 11 & 36,67 & 6 & 20,00 \\
\hline CN & 10 & 33,33 & 1 & 3,33 & 4 & 13,33 & 6 & 20,00 & 6 & 20,00 \\
\hline $\mathbf{N}$ & 4 & 13,33 & 1 & 3,33 & 0 & 0,00 & 2 & 6,67 & 3 & 10,00 \\
\hline Total & 30 & 100,00 & 30 & 100,00 & 30 & 100,00 & 30 & 100,00 & 30 & 100,00 \\
\hline Media ítem & \multicolumn{2}{|c|}{2,67} & \multicolumn{2}{|c|}{3,47} & \multicolumn{2}{|c|}{3,47} & \multicolumn{2}{|c|}{3,13} & \multicolumn{2}{|c|}{3,23} \\
\hline Categoría & \multicolumn{2}{|c|}{$\begin{array}{l}\text { Moderado } \\
\text { uso }\end{array}$} & \multicolumn{2}{|c|}{ Alto uso } & \multicolumn{2}{|c|}{ Alto uso } & \multicolumn{2}{|c|}{$\begin{array}{l}\text { Moderado } \\
\text { uso }\end{array}$} & \multicolumn{2}{|c|}{$\begin{array}{l}\text { Moderado } \\
\text { uso }\end{array}$} \\
\hline $\begin{array}{l}\text { Media } \\
\text { Indicador }\end{array}$ & \multicolumn{10}{|c|}{3,19} \\
\hline Categoría & \multicolumn{10}{|c|}{ Moderado uso } \\
\hline Desviación & \multicolumn{10}{|c|}{1,02} \\
\hline Categoría & \multicolumn{10}{|c|}{ Moderada dispersión / Moderada confiabilidad } \\
\hline
\end{tabular}

Por otro lado, al analizar los resultados obtenidos para cada uno de los ítems del indicador, cabe destacar que para el caso específico de los reactivos 1,4 y 5 , relativos a la frecuencia de fallas de los equipos y si llevan registros de la confiabilidad y de las fallas, éstos obtuvieron medias aritméticas de 2,67; 3,13 y 3,23 ubicándose, de acuerdo a la percepción de los encuestados, dentro de la categoría de moderado uso, según la interpretación del baremo correspondiente.

La tabla 3 muestra los resultados alcanzados por el indicador mantenibilidad de la dimensión indicadores de la gestión de mantenimiento, el cual obtuvo una media aritmética de 3,30 evidenciando que, según la opinión de los encuestados, este indicador de gestión presenta una un moderado uso en la gestión de mantenimiento que realizan las empresas de servicio petrolero de la Costa Oriental del Lago analizadas. De igual modo, éste indicador obtuvo una desviación estándar de 1,05 señalando una moderada dispersión de las respuestas, así como una moderada confiabilidad de las mismas. 
Tabla 3. Resultados indicador mantenibilidad.

\begin{tabular}{|c|c|c|c|c|c|c|c|c|c|c|}
\hline № ítem & & 1 & & 2 & & 3 & & 4 & & 5 \\
\hline $\begin{array}{l}\text { Descripción } \\
\text { Ítem }\end{array}$ & $\begin{array}{l}\text { La v } \\
\text { de re } \\
\text { ante } \\
\text { corr } \\
\text { a las } \\
\text { nece } \\
\text { de la } \\
\text { emp }\end{array}$ & $\begin{array}{l}\text { ocidad } \\
\text { puesta } \\
\text { a fallas } \\
\text { sponde } \\
\text { idades } \\
\text { esa. }\end{array}$ & $\begin{array}{l}\text { Se t } \\
\text { prol } \\
\text { tipo } \\
\text { falla } \\
\text { equ }\end{array}$ & $\begin{array}{l}\text { ene un } \\
\text { nedio de } \\
\text { de } \\
\text { s por } \\
\text { po. }\end{array}$ & $\begin{array}{l}\text { Deter } \\
\text { proba } \\
\text { que u } \\
\text { estad } \\
\text { sea re } \\
\text { a su c } \\
\text { opera } \\
\text { perío } \\
\text { tiemp } \\
\text { adect }\end{array}$ & $\begin{array}{l}\text { Ina la } \\
\text { lidad de } \\
\text { equipo en } \\
\text { le falla } \\
\text { ablecido } \\
\text { dición de } \\
\text { ón en un } \\
\text { de } \\
\text { lo. }\end{array}$ & $\begin{array}{l}\text { Se u } \\
\text { tiem } \\
\text { prol } \\
\text { par } \\
\text { las } \\
\text { rep }\end{array}$ & $\begin{array}{l}\text { liza un } \\
\text { oo } \\
\text { edio } \\
\text { realizar } \\
\text { aciones. }\end{array}$ & $\begin{array}{l}\text { Se cu } \\
\text { sufici } \\
\text { perso } \\
\text { cubri } \\
\text { neces } \\
\text { mant }\end{array}$ & $\begin{array}{l}\text { ta con } \\
\text { ate } \\
\text { al para } \\
\text { las } \\
\text { lades de } \\
\text { imiento. }\end{array}$ \\
\hline Opción & $\mathbf{F a}$ & Fr & $\mathbf{F a}$ & Fr & $\mathbf{F a}$ & $\mathrm{Fr}$ & $\mathbf{F a}$ & $\mathrm{Fr}$ & $\mathbf{F a}$ & $\mathrm{Fr}$ \\
\hline S & 5 & 16,67 & 4 & 13,33 & 3 & 10,00 & 3 & 10,00 & 4 & 13,33 \\
\hline CS & 12 & 40,00 & 7 & 23,33 & 10 & 33,33 & 12 & 40,00 & 6 & 20,00 \\
\hline $\mathbf{A V}$ & 10 & 33,33 & 9 & 30,00 & 12 & 40,00 & 5 & 16,67 & 10 & 33,33 \\
\hline CN & 3 & 10,00 & 8 & 26,67 & 3 & 10,00 & 9 & 30,00 & 9 & 30,00 \\
\hline $\mathbf{N}$ & 0 & 0,00 & 2 & 6,67 & 2 & 6,67 & 1 & 3,33 & 1 & 3,33 \\
\hline Total & 30 & 100,00 & 30 & 100,00 & 30 & 100,00 & 30 & 100,00 & 30 & 100,00 \\
\hline Media ítem & &, 63 & & 3,10 & & 30 & & 3,23 & & 63 \\
\hline Categoría & & to uso & & $\begin{array}{l}\text { derado } \\
\text { uso }\end{array}$ & Moc & ado uso & & $\begin{array}{l}\text { derado } \\
\text { uso }\end{array}$ & & o uso \\
\hline $\begin{array}{l}\text { Media } \\
\text { Indicador }\end{array}$ & & & & & & 3,30 & & & & \\
\hline Categoría & & & & & & rado uso & & & & \\
\hline Desviación & & & & & & 1,05 & & & & \\
\hline Categoría & & & & derada d & spersic & / Moderac & con & abilidad & & \\
\hline
\end{tabular}

Al analizar en detalle el indicador, se puede apreciar que para los reactivos 1 y 5 , las medias alcanzadas son de 3,63, para ambos casos, ubicando a las actividades involucradas en dichos ítems en la categoría de alta uso. Para el caso de los ítems 2, 3 y 4 las medias obtenidas por los reactivos es de 3,$10 ; 3,30 ;$ y 3,23 ; las cuales los ubican dentro de la categoría de moderado uso en la gestión de mantenimiento que se analiza.

Es de hacer notar que las concentraciones de respuesta de los encuestados no se perfilan a una alternativa, situación que se vislumbra también en los tres indicadores de esta dimensión, mostrándose los resultados con cierta preferencia por las opciones casi siempre y algunas veces, esto explica que la media de los indicadores, se ubiquen en la categoría de moderado uso.

La tabla 4 presenta los resultados logrados para el indicador costos se cual obtuvo una media aritmética de 3,27 reflejado que de acuerdo a la percepción de los encuestados, el indicador de gestión costos en la gestión que adelantan las empresas demuestran un moderado uso, respecto a la manera en que se utiliza el mismo. 
Por otro lado, este indicador presenta una desviación estándar de 1,18 señalando una moderada dispersión de las respuestas dadas por los encuestados, así como una moderada confiabilidad de éstas.

Tabla 4. Resultados indicador costos

\begin{tabular}{|c|c|c|c|c|c|c|c|c|c|c|c|}
\hline № ítem & & 1 & \multicolumn{2}{|c|}{2} & 3 & & \multicolumn{3}{|c|}{4} & \multicolumn{2}{|c|}{5} \\
\hline $\begin{array}{l}\text { Descripción } \\
\text { Ítem }\end{array}$ & \multicolumn{2}{|c|}{$\begin{array}{l}\text { Utiliza } \\
\text { indicadores de } \\
\text { costo para } \\
\text { medir la } \\
\text { gestión de } \\
\text { mantenimiento. }\end{array}$} & \multicolumn{2}{|c|}{$\begin{array}{l}\text { El uso de los } \\
\text { recursos para } \\
\text { mantenimiento } \\
\text { asegura el } \\
\text { menor costo } \\
\text { por actividad. }\end{array}$} & $\begin{array}{l}\text { Maneja } \\
\text { índices } \\
\text { econón } \\
\text { que } \\
\text { permit } \\
\text { medir l } \\
\text { eficien } \\
\text { en el us } \\
\text { los rect } \\
\text { utilizad }\end{array}$ & $\begin{array}{l}\text { cos } \\
\text { de } \\
\text { sos } \\
\text { s. }\end{array}$ & \multicolumn{3}{|c|}{$\begin{array}{ll}\text { El } & \mathrm{S} \\
\text { presupuesto } & \mathrm{p} \\
\text { es acorde con } & \mathrm{l} \\
\text { lo requerido } & \mathrm{f} \\
\text { en las } & \mathrm{p} \\
\text { requisiciones } & \mathrm{r} \\
\text { programadas. } & \mathrm{n}\end{array}$} & \multicolumn{2}{|c|}{$\begin{array}{l}\text { Se revisan } \\
\text { periódicamente } \\
\text { los costos con el } \\
\text { fin de establecer } \\
\text { programas de } \\
\text { reducción de los } \\
\text { mismos. }\end{array}$} \\
\hline Opción & $\mathbf{F a}$ & Fr & $\mathbf{F a}$ & Fr & $\mathbf{F a}$ & \multicolumn{2}{|l|}{ Fr } & $\mathbf{F a}$ & Fr & $\mathbf{F a}$ & Fr \\
\hline$S$ & 6 & 20,00 & 4 & 13,33 & 6 & \multicolumn{2}{|c|}{20,00} & 3 & 10,00 & 6 & 20,00 \\
\hline CS & 10 & 33,33 & 9 & 30,00 & 9 & \multicolumn{2}{|c|}{30,00} & 7 & 23,33 & 5 & 16,67 \\
\hline AV & 6 & 20,00 & 11 & 36,67 & 5 & \multicolumn{2}{|c|}{16,67} & 14 & 46,67 & 11 & 36,67 \\
\hline CN & 5 & 16,67 & 4 & 13,33 & 8 & \multicolumn{2}{|c|}{26,67} & 4 & 13,33 & 5 & 16,67 \\
\hline $\mathbf{N}$ & 3 & 10,00 & 2 & 6,67 & 2 & \multicolumn{2}{|c|}{6,67} & 2 & 6,67 & 3 & 10,00 \\
\hline Total & 30 & 100,00 & 30 & 100,00 & 30 & \multicolumn{2}{|c|}{100,00} & 30 & 100,00 & 30 & 100,00 \\
\hline Media ítem & \multicolumn{2}{|c|}{3,37} & \multicolumn{2}{|c|}{3,30} & \multicolumn{2}{|c|}{3,30} & \multicolumn{3}{|c|}{3,17} & \multicolumn{2}{|c|}{3,20} \\
\hline Categoría & \multicolumn{2}{|c|}{ Moderado uso } & \multicolumn{2}{|c|}{ Moderado uso } & $\begin{array}{r}\text { Moder } \\
\text { us }\end{array}$ & & \multicolumn{3}{|c|}{ Moderado uso } & \multicolumn{2}{|c|}{ Moderado uso } \\
\hline $\begin{array}{l}\text { Media } \\
\text { Indicador }\end{array}$ & \multicolumn{11}{|c|}{3,27} \\
\hline Categoría & \multicolumn{11}{|c|}{ Moderado uso } \\
\hline Desviación & \multicolumn{11}{|c|}{1,18} \\
\hline Categoría & \multicolumn{11}{|c|}{ Moderada dispersión / Moderada confiabilidad } \\
\hline
\end{tabular}

Al observar detalladamente los resultados alcanzados por cada ítem del indicador, puede apreciarse que todos los reactivos, con medias aritméticas de 3,37, 3,$30 ; 3,30 ; 3,17 ;$ y 3,20 , respectivamente, se ubican, según la opinión de los encuestados, dentro de la categoría de moderado uso, es decir, se utiliza de manera moderada todas las actividades, consideradas en el cuestionario, que involucran la medición del indicador costos en la gestión de mantenimiento que se analiza. 
Nótese que en todos los ítems, las respuestas no tuvieron concentración hacia una escala en particular, dada la moderada dispersión de las mismas. Por ejemplo, al observar el ítem 1 el comportamiento de las respuestas fue $20 \%$ en la opción siempre, $33,33 \%$ casi siempre, $20 \%$ algunas veces, $16,67 \%$ casi nunca, y $10 \%$ nunca, no mostrando homogeneidad de criterio para sus respuestas, por parte de los sujetos encuestados. Situaciones similares ocurren en el resto de los reactivos aplicados.

Una vez analizados e interpretados los resultados obtenidos para los indicadores de gestión de mantenimiento, a continuación se presenta la tabla 5 , donde se muestra, en síntesis, el comportamiento alcanzado por la misma, a la vez que sirve de base para la realización de la discusión de dichos resultados.

Tabla 5. Resultados de los indicadores de gestión de mantenimiento en empresas de Servicio petrolero de la Costa Oriental del Lago

\begin{tabular}{lccl}
\hline Indicador & $\overline{\boldsymbol{X}}$ & $\mathbf{S}$ & Categorías \\
\hline Disponibilidad & 3,35 & 0,99 & Moderado uso/ Moderada dispersión \\
Confiabilidad & 3,19 & 1,02 & Moderado uso/ Moderada dispersión \\
Mantenibilidad & 3,30 & 1,05 & Moderado uso/ Moderada dispersión \\
Costos & 3,27 & 1,18 & Moderado uso/ Moderada dispersión \\
\hline \multicolumn{1}{c}{ Resumen dimensión } & $\mathbf{3 , 2 7}$ & $\mathbf{1 , 0 6}$ & Moderado uso/ Moderada dispersión \\
\hline
\end{tabular}

La tabla 5, muestra la distribución de las medias y la desviación estándar que describen el comportamiento de los indicadores de la gestión de mantenimiento. Se evidencia que, según la percepción de los encuestados, en las empresas de servicio petrolero de la Costa Oriental del Lago se ostenta un moderado uso de los indicadores de la gestión de mantenimiento en la medición de su desempeño, al obtener una media aritmética de 3,27. Exhibiendo, a la vez, una moderada dispersión de las respuestas dadas por los encuestados y una moderada confiabilidad de la misma, al obtener una desviación estándar de 1,06; en función de la interpretación de los baremos correspondientes.

De acuerdo a los resultados reflejados en la tabla, todos los indicadores de gestión, considerados en esta investigación para medir el desempeño de la gestión de mantenimiento, se implementan en las empresas analizadas, sin embargo, todos reflejan una condición de moderado uso. Situación que se ratifica con las medias aritméticas obtenidas por los indicadores involucrados, posicionando a la dimensión indicadores de la gestión de mantenimiento, dentro de la categoría de un moderado uso.

El análisis precedente, presenta discrepancias con la teoría de los autores, entre los que destacan Jiménez y Milano (2006), cuando expresa que los indicadores que se pueden llevar en la gestión de mantenimiento, se implementan para medir la gestión de mantenimiento de los sistemas productivos, los cuales son utilizados para cumplir con las metas definidas en un plan 
de trabajo generalmente realizado anualmente. Dicho esto, las empresas de servicio petrolero, al presentar un moderado uso de estos indicadores, difícilmente podrán ser efectivas en el logro de sus metas, y por ende de los objetivos organizacionales.

De igual manera, esta situación reflejada por los resultados valida medianamente lo expuesto por Nava (2009), Leal y Zambrano (2011), Hernández y Navarrete (2005), quienes define los indicadores de la gestión de mantenimiento como índices de control de mantenimiento, indicadores o medidores de evaluación, los cuales con su uso permiten a cualquier gestión de mantenimiento tener un señalamiento del comportamiento o de la efectividad del sistema total. Cabe decir, que al ser usados de manera moderada limita su efectividad para medir el desempeño de la gestión, lo cual, a su vez, limita la utilización de acciones correctivas.

Adicional a lo anterior, si se compara con los postulados de Leal y Zambrano (2011), se hallan ciertas incongruencias entre lo que plantea la teoría y lo que se viene haciendo en esta gestión que se analiza, en cuanto a que consideran que la gestión de mantenimiento debe estar en una continua evaluación para lograr la optimización del mismo, y una forma de encontrarlo es por medio de la generación, aplicación y evaluación de indicadores de gestión. Así las cosas, esto permite llevar un control que oriente a la empresa para minimizar los tiempos de entrega, aumentar la satisfacción del cliente y que por ende traerá como consecuencia mayor margen de ganancia o beneficio a través de mínimos costos operacionales.
En el caso del indicador disponibilidad, el cual muestra un moderado uso, esto pudiera ser desfavorable, dado que según Duffuaa y col. (2014), Souris (2010), García (2013) y Ferrer (2009) al no medir de manera continua el indicador disponibilidad se corre el riesgo de no conocer si los equipos e instalaciones están totalmente en capacidad para llevar a cabo con éxito la función requerida en un momento específico o durante un período de tiempo específico, es decir, no se tendría la certeza de la disponibilidad funcional del equipo o instalación en los requerimientos de los sistemas productivos.

En esta perspectiva, para el indicador confiabilidad, el cual presenta también moderado uso, se tiene el riesgo de estar operando equipos o sistemas que podrían ser no confiables. Situación está que no concuerda con los postulados de Duffuaa y col. (2014), Rodríguez (2008), Tavares (2006), Hernández y Navarrete (2005) para quienes la confiabilidad es la probabilidad de que un equipo o sistema de producción desarrolle su función, bajo condiciones específicas y durante un período de tiempo determinado, para lo cual es imprescindible monitorear su funcionamiento mediante los indicadores de gestión de confiabilidad o fiabilidad.

De esta manera, la gestión de mantenimiento en las empresas bajo estudio, presentan ciertas debilidades en cuanto al uso de este indicador, lo cual puede limitar el que los equipos o sistemas estén operando sin ningún contratiempo, al ser requerido por el sistema de producción.

En cuanto al indicador mantenibilidad, que igual presenta moderado uso, indicando que hay una frecuencia media en la utilización del indicador, se evidencia, de 
igual manera que en los dos anteriores, discrepancias en cuanto al deber ser. Como lo explican Mesa y col. (2006), Torres (2010), Nava (2009), Rodríguez (2008) y García (2011), este indicador permite conocer la probabilidad de que el equipo sea restaurado a sus condiciones normales de funcionabilidad, luego de la aparición de la falla, bajo condiciones preestablecidas de mantenimiento. Visto así, el resultado de moderada frecuencia, se indica la necesidad de fortalecer el empleo de este indicador, recordando que a mayor mantenibilidad menor tiempo de ejecución de los trabajos y viceversa.

Finalmente, se tiene el moderado uso del indicador costos, como consecuencia de una frecuencia media en la utilización de este indicador en la gestión de mantenimiento que se analiza. Debe destacarse la importancia de los indicadores de costos, dado que hoy en día los mismos se han convertido en una prioridad de control, tal como lo señalan Rodríguez (2008), Molina (2015), y Duffuaa y col. (2014), es un índice económico que permite el seguimiento de la evolución de los resultados internos y cierta comparación del mantenimiento entre diferentes plantas.

Adicionalmente, dado el moderado uso de los indicadores en la gestión de mantenimiento en las empresas analizadas, se debe reflexionar en aspectos como los que aborda Martínez (2007), para quien desde el punto de vista de la organización de mantenimiento el reporte de los costos debe servir a los siguientes requisitos específicos: supervisión del personal que labora en los mantenimientos, la cual necesita medir su efectividad; la gerencia de mantenimiento requiere observar la tendencia de los costos, con el detalle necesario para atender las áreas de atención especial y así lograr reducir los costos de mantenimiento, sin afectar la calidad y la producción; y por último la gerencia general debe conocer los costos relacionados con el mantenimiento, los cuales son esenciales para calcular los costos de producción.

En síntesis, los resultados alcanzados por los indicadores de la gestión de mantenimiento, revelan un moderado uso de los indicadores considerados en esta investigación, lo cual devela la necesidad de revisar detalladamente las ventajas $\mathrm{y}$ desventajas del uso frecuente de cada uno de ellos por parte de las empresas de servicio petrolero de la Costa Oriental del Lago involucradas, de manera que estas puedan desarrollar o afianzar un conjunto de indicadores que les permitan medir la efectividad de su gestión de mantenimiento, que les asegure la excelencia en la gestión.

\section{CONCLUSIONES}

En el ámbito empresarial se conoce como indicador de gestión a aquel dato que refleja cuáles fueron las consecuencias de acciones tomadas en el pasado en el marco de una organización. La idea es que estos indicadores sienten las bases para acciones a tomar en el presente y en el futuro. Es importante que los indicadores de gestión reflejen datos veraces y fiables, ya que el análisis de la situación, de otra manera, no es correcto. Por otra parte, si los indicadores son ambiguos, la interpretación será complicada. Lo que permite un indicador de gestión es determinar si un proyecto o una organización están siendo exitosos o si están cumpliendo con los objetivos.

En función de los resultados obtenidos los indicadores presentan un moderado uso 
en la gestión de mantenimiento que se analiza. Evidentemente esto denota debilidades de la gestión, al no medir su desempeño para conocer desviaciones entre lo planificado y lo realizado, reflejando cuáles fueron las consecuencias de acciones tomadas en el pasado en el marco del mantenimiento. La idea es que estos indicadores sienten las bases para tomar acciones en el área de mantenimiento en el presente y en el futuro.

Finalmente, cabe resaltar entonces que la gestión de mantenimiento en las empresas de servicio petrolero, deben revisar sus estrategias en cuanto al uso más frecuente de los indicadores que se pueden llevar en la gestión de mantenimiento, los cuales deben ser utilizados para cumplir con las metas definidas en el plan de trabajo. Dicho esto, las empresas analizadas, al presentar un moderado uso de estos indicadores, difícilmente podrán ser efectivas en el logro de sus metas, y por ende de los objetivos organizacionales.

\section{REFERENCIAS}

Arias, F. (2016). El proyecto de investigación: Introducción a la metodología científica. Caracas: Editorial Episteme

Duffuaa, S., Raouf, A. y Campbell, J. (2014). Sistemas de Mantenimiento. Planificación y Control. México: Editorial Limusa

Ferrer, A. (2009). Gerencia de activos. Beneficios de la gerencia de activos. Colombia: Ediciones ECOE

García, R. (2013). Planificación estratégica aplicada al mantenimiento. México: Editorial Mc-Graw Hill

Heredia, N. (2013). Gerencia de compras: La nueva estrategia competitiva. Bogotá: Ediciones ECOE

Hernández, E. y Navarrete, E. (2005). Sistema de cálculo de indicadores para el mantenimiento. Revista Club de mantenimiento. Año 2. № 6. P. 15-20. Brasil

Hernández, R; Fernández, C; y Baptista, P. (2014). Metodología de la investigación. México: McGraw-Hill/ Interamericana Editores. S.A. de C.V

Hurtado, J. (2015) Metodología de la Investigación. Caracas: Editorial Sypal

Heredia, N. (2013). Gerencia de compras: La nueva estrategia competitiva. Bogotá: Ediciones ECOE

Kaplan, R. y Norton, D. (2002). Cuadro de Mando Integral (The Balance Scorecard). España: Ediciones Gestión 2000

Jiménez, K. y Milano, T. (2006). Planificación y gestión del mantenimiento industrial. Un enfoque estratégico y operativo. Caracas: Editorial Panapo

Leal, S. y Zambrano, S. (2011). Índices e Indicadores de Gestión de Mantenimiento en las Pymes del Estado Táchira. 3er Congreso Uruguayo de Mantenimiento. Recuperado el 06 de mayo de 2020, de: http://www.uruman.org/3er_congreso_ docs/trabajos_tecnicos/Articulo $\% 20 \% 2$ 0URUM AN\%20-\%20Indicadores.pdf

Martínez, L. (2007). Organización y planificación de sistemas de mantenimiento. Centro de altos estudios gerenciales. Caracas: Instituto Superior de Investigación y Desarrollo.

Mesa, D. Ortiz, Y. y Pinzón, M. (2006). La confiabilidad, la disponibilidad y la mantenibilidad. Disciplinas modernas aplicadas al mantenimiento. En revista Scientia et Technica. La universidad pedagógica de Pereira. Volumen 1, № 30 Recuperado el 20 de mayo de 2020, de http://www.redalyc.org/pdf/849/8492 0491036.pdf

Molina, A. (2015) Aplicación Práctica de la Teoría de Mantenimiento. Mérida: Consejo de Publicaciones, Universidad Los Andes 
Mora, A. (2011). Mantenimiento. Planeación, ejecución y control. México: Editorial Alfa-Omega Grupo Editor, S.A. México D.F

Nava, J. (2009). Aplicación práctica de la teoría de mantenimiento. Editorial Consejo de publicaciones de la Universidad de los Andes. Mérida. Venezuela.

Rodríguez, J. (2008). Gestión de mantenimiento. Introducción a la teoría del mantenimiento. Casos empresariales. Recuperado el 05 de junio de 2020, de: http://www.scribd.com/doc/7497765/ gestion-del-mantenimiento

Souris, J. (2010). El mantenimiento, fuente de beneficios. Madrid: Ediciones Díaz de Santos

Tavares, L. (2006). Modelos de mantenimiento. México: Editorial Limusa

Torres, L. (2010). Mantenimiento, su implementación y gestión. Argentina: Editorial Universitas 